\title{
Interplay of the Glass Transition and the Liquid-Liquid Phase Transition in Water
}

SUBJECT AREAS: CHEMICAL PHYSICS

PHYSICAL CHEMISTRY

CHEMISTRY

PHYSICS

Received

27 February 2012

Accepted

18 April 2012

Published

1 May 2012

Correspondence and requests for materials should be addressed to N.G.

(ngiovambattista@ brooklyn.cuny.edu) or T.L. (thomas.loerting@ vibk.ac.at) or F.W.S. (fstarr@wesleyan.edu)
Nicolas Giovambattista' ${ }^{1}$ Thomas Loerting ${ }^{2}$, Boris R. Lukanov ${ }^{3}$ \& Francis W. Starr ${ }^{3}$
'Department of Physics, Brooklyn College of the City University of New York, Brooklyn, NY 1 1210, USA, ${ }^{2}$ Institute of Physical Chemistry, University of Innsbruck, Innrain 52a, A-6020 Innsbruck, Austria, ${ }^{3}$ Department of Physics, Wesleyan University, Middletown, CT 06459, USA
Water has multiple glassy states, often called amorphous ices. Low-density (LDA) and high-density (HDA) amorphous ice are separated by a dramatic, first-order like phase transition. It has been argued that the LDA-HDA transformation connects to a first-order liquid-liquid phase transition (LLPT) above the glass transition temperature $T_{g}$. Direct experimental evidence of the LLPT is challenging to obtain, since the LLPT occurs at conditions where water rapidly crystallizes. In this work, we explore the implications of a LLPT on the pressure dependence of $T_{g}(P)$ for LDA and HDA by performing computer simulations of two water models - one with a LLPT, and one without. In the absence of a LLPT, $T_{g}(P)$ for all glasses nearly coincide. When there is a LLPT, different glasses exhibit dramatically different $T_{g}(P)$ which are directly linked with the LLPT. Available experimental data for $T_{g}(P)$ are only consistent with the scenario including a LLPT.

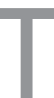

he ubiquity and biological importance of water, as well as its numerous puzzling anomalous properties, have sparked a long history of water research. Over the last 40 years, there have been several frameworks proposed to explain the unusual behavior of liquid water ${ }^{1-3}$, most notably the liquid-liquid critical point (LLCP) hypothesis $^{4,5}$, the stability limit conjecture ${ }^{6}$, and the singularity-free scenario ${ }^{7}$. Most relevant to this work is the LLCP hypothesis, which proposes that the unusual behavior of liquid water can be explained by the existence of two thermodynamically distinct phases - a low-density liquid (LDL) and high-density liquid (HDL) - below a critical temperature $T_{c}$. In this scenario, LDL and HDL are separated by a liquid-liquid phase transition (LLPT) ending at a LLCP. While there are many simulations of atomistic water models ${ }^{4,8-17}$ and other tetrahedrally coordinated liquids ${ }^{18-21}$ that support this possibility, direct experimental confirmation of this proposal has been prevented by the nucleation of crystalline ice. Recent experiments of water confined at the nanoscale (e.g., Ref.22), where crystallization can be suppressed, support the view that two liquids, LDL and HDL, exist at low temperatures. Although encouraging, it is not clear at present what role the confining surfaces play on water phase behavior (see, e.g., Ref.23).

Some of the strongest experimental evidence suggesting a liquid-liquid transition in water are obtained from glassy water. Specifically, experiments show the presence of two distinct glassy states, low-density (LDA) and high-density amorphous (HDA) ice, which can be reversibly interconverted by the application (or removal) of pressure $^{24-28}$. Very recently, Winkel et al. observed the transformation from high- to low-density water by removal of pressure at $140 \mathrm{~K}$, and suggested it to be of first-order in the ultraviscous liquid domain just above $T_{g}{ }^{29}$. In the context of the LLCP hypothesis, LDA and HDA are envisioned as the glass counterparts of LDL and HDL, respectively. Unambiguous evidence that LDA and HDA are thermodynamically distinct and that they are thermodynamically continuously connected with LDL and HDL, respectively, would imply the existence of polymorphism in liquid water and strongly support the existence of a LLCP. Unfortunately, the rapid crystallization and non-equilibrium nature of glassy water complicates elucidating the relationship between the equilibrium liquid and LDA/HDA, and hence complicates determining whether two liquid phases exist in water.

A possible way to overcome this challenge is to take advantage of the fact that, at the glass transition temperature $T_{g}$, the ergodic liquid is restored (if only briefly before crystallization occurs ${ }^{30-32}$ ). Consequently, the pressure dependence of $T_{g}(P)$ provides valuable information on the thermodynamics of the liquid state. This is conceptually similar to using the metastable melting loci to extract information about the liquid state, an approach developed by Mishima and Stanley $^{5}$. If there are thermodynamically distinct liquid states (which have a discontinuous change in dynamics at the LLPT) there should be a discontinuous change in the locus $T_{g}(P)$ obtained from quenching the liquid under pressure. Additionally, pressure treated glassy states are expected to have dramatically different $T_{g}(P)^{33}$. Hence, $T_{g}(P)$ may offer a direct indicator of a LLPT. The primary obstacle to examining this approach experimentally is maintaining specific 
large pressure and avoiding crystallization during thermal studies of various glasses, although some researchers ${ }^{29,34-36}$ have begun to overcome these problems.

Motivated by these considerations, here we examine the behavior of the locus of $T_{g}(P)$ for the amorphous ices using computer simulations of two models of water - one that has a highly accessible LLPT (the ST2 model) $)^{4,8,11,12,17}$, and one that does not (the SPC/E model) $)^{37-}$ ${ }^{39}$. We select these models to provide clearly contrasting behavior, rather than for their accuracy (or lack thereof) in reproducing quantitative experimental behavior of water. Our simulations show that, in the absence of a LLPT (the SPC/E model), the glass transition loci $T_{g}(P)$ of HDA and LDA are nearly indistinguishable, and that $T_{g}(P)$ varies smoothly with pressure. In marked contrast, when there is a LLPT (the ST2 model), the $T_{g}(P)$ loci of LDA and HDA have opposite pressure dependences. By also evaluating $T_{g}(P)$ from glasses obtained by hyperquenching the equilibrium liquid under pressure, we show that this $T_{g}(P)$ (which is directly related to liquid states) roughly coincides with that of LDA at low pressure, and with that of HDA at high pressure; this $T_{g}(P)$ exhibits a sharp change from LDA- to HDA-like behavior at the intersection with the known LLPT line. The qualitative differences between the results obtained with the SPC/E and ST2 models provide a template to interpret experiments measuring $T_{g}(P)$ from amorphous ices, and hence to distinguish whether or not the behavior of water is indicative of an LLPT. We also discuss the available experimental data, which are qualitatively consistent with the LLPT scenario shown by the ST2 model, and inconsistent with the SPC/E model; further experiments are needed for confirmation.

\section{Results}

Glassy States under Consideration. The many glassy states of water can be broadly categorized into low- and high-density forms. The properties of the glassy states of the SPC/E and ST2 models have been previously examined, and it has been shown that both models qualitatively reproduce the LDA and HDA states ${ }^{40-45}$. In the following sections, we will examine hyperquenched glassy water (HGW) under compression, and HDA under both compression and decompression; we also examine LDA, obtained by heating HDA at low pressure. We follow procedures similar to those used in experiments ${ }^{24,31,46}$ to prepare these states, although the cooling/ heating and compression/decompression rates accessible in simulations are significantly faster than those used in experiments. See the Methods section for a complete description of the preparation of these glasses.

For the ST2 model case, we also examine glasses formed by direct hyperquenches of the liquid at different pressures. Since these hyperquenched glasses (HQG) are obtained by quenching the liquid under pressure, they are directly related to the liquid state at the same pressure. Note that for the particular case of $P=0.1 \mathrm{MPa}$ (and only for this pressure) HQG is the same as HGW. Rapid quenching of bulk samples under pressure is experimentally very challenging; this approach has been pursued experimentally only for $\mu \mathrm{m}$ size droplets of emulsified water where crystallization is suppressed ${ }^{34}$.

For each glass we form, we evaluate the glass transition temperature locus, $T_{g}(P)$. For a given glass at pressure $P$, we determine $T_{g}(P)$ following a procedure analogous to that employed in differential scanning calorimetry (DSC) experiments (see Methods section). The data presented are an average over twenty independent simulations for each glass.

Glass Transition in the Absence of a Liquid-Liquid Phase Transition. We first consider the behavior of the various glasses in the SPC/ E model, where there is no interference of an LLPT to complicate the behavior of $T_{g}(P)$. Figure 1 shows the $T_{g}(P)$ loci for (i) compressed HGW, $T_{g}^{\mathrm{HGW}}(P)$, (ii) HDA obtained during compression of ice $\mathrm{I}_{h}$, and (iii) decompressed HDA, $T_{g}^{\mathrm{HDA}}(P)$ (again, averaged over twenty independent simulations). All glasses are heated from an initial temperature of $T=80 \mathrm{~K}$.

Remarkably, Fig. 1 shows that in the absence of an LLPT, the $T_{g}$ loci are almost completely independent of the starting glassy state, i.e., $T_{g}^{\mathrm{HGW}}(P)$ and $T_{g}^{\mathrm{HDA}}(P)$ (under compression or decompression) all roughly collapse onto a single $T_{g}(P)$ locus. This is particularly surprising given that the initial density of these glasses can differ by more than $20 \%$, such as in the case of compressed HGW and decompressed HDA at $P \lesssim 1200 \mathrm{MPa}$ (see Methods section to compare the initial densities). However, it should be noted that, while $T_{g}(P)$ of the various glasses are nearly the same, the behavior of $c_{P}(T)$ (from which $T_{g}(P)$ is determined) and $\rho(T)$ upon heating differs significantly for $T<T_{g}$ (Fig. 2).

Interestingly, Fig. 1 shows that $T_{g}(P)$ has a minimum at $P \approx$ $300 \mathrm{MPa}$. Such a minimum occurs at the intersection of the $T_{g}(P)$ locus and the maximum diffusivity line ( $D_{\max }$-line). The $D_{\max }$-line is defined as the set of pressures at which the diffusion coefficient $D$ exhibits a maximum upon isothermal compression; its presence is a consequence of the well-known anomalous pressure-dependence of water diffusivity. The coincidence of the $T_{g}(P)$ minimum and the $D_{\text {max }}$-line is a reassuring consistency of the definition of $T_{g}$ employed in this work, since we define $T_{g}$ by a thermodynamic (rather than dynamic) criterion.

We can quantitatively explain the observed relation between the minimum of $T_{g}(P)$ and the $D_{\max }$ locus by considering the diffusive relaxation time $\tau \propto(D / T)^{-147}$ as an explicit function of $T$ and $P$. Consider the total differential

$$
d \tau(T, P)=\left(\frac{\partial \tau}{\partial T}\right)_{P} d T+\left(\frac{\partial \tau}{\partial P}\right)_{T} d P .
$$

Experimentally, $T_{g}(P)$ is often defined as the temperature at which $\tau(T, P)$ reaches a fixed value (typically $100 \mathrm{~s}$ ) for a given cooling/ heating rate ${ }^{48}$. Since all glasses in our simulations are formed using the same cooling/heating rate at all pressures, it follows that $\tau$ should be constant along the $T_{g}(P)$ locus, and thus $d \tau=0$ along $T_{g}(P)$. Therefore, rearranging eq. (1),

$$
\frac{d T_{g}(P)}{d P}=-\left(\frac{\partial \tau}{\partial P}\right)_{T} /\left(\frac{\partial \tau}{\partial T}\right)_{P}
$$

along the $T_{g}(P)$ locus. The denominator $(\partial \tau / \partial T)_{P}<0$ and finite, since $\tau$ is a monotonically decreasing function of $T$. Hence, $d T_{g}(P) / d P=0$ (where $T_{g}(P)$ is minimum) only if the numerator $(\partial \tau / \partial P)_{T}=0$ at $T_{g}$, and hence $\tau$ (and correspondingly $D$ ) must be an extremum as a

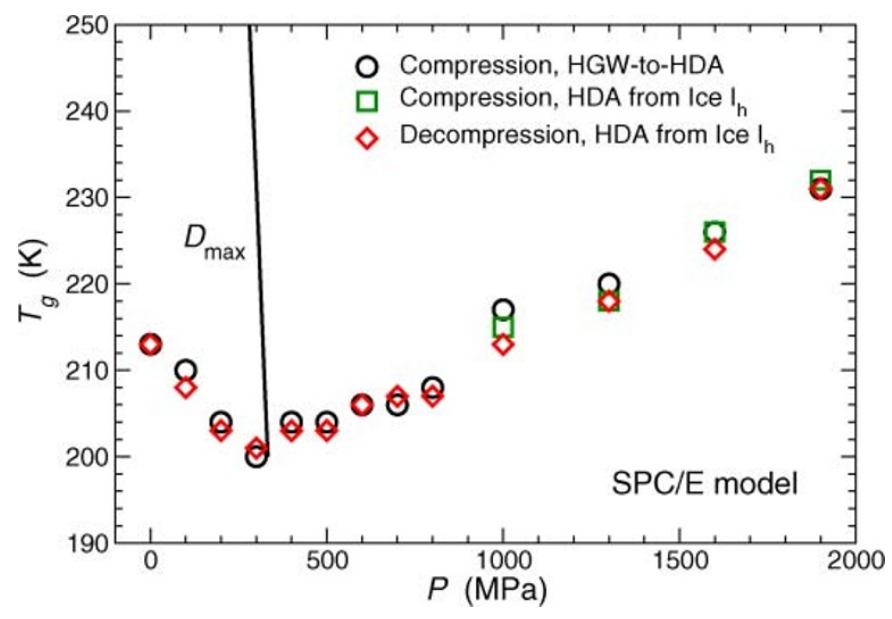

Figure $1 \mid$ Pressure-dependence of the glass transition temperature $T_{g}(P)$ for the SPC/E model. The behavior of $T_{g}(P)$ is nearly independent of the glass preparation procedure. At pressures $P \gtrsim 1200 \mathrm{MPa}$, compression of HGW yields HDA (see methods section on preparation of glasses). The maximum diffusivity line ( $D_{\max }-$ line) is taken from Ref.38. 

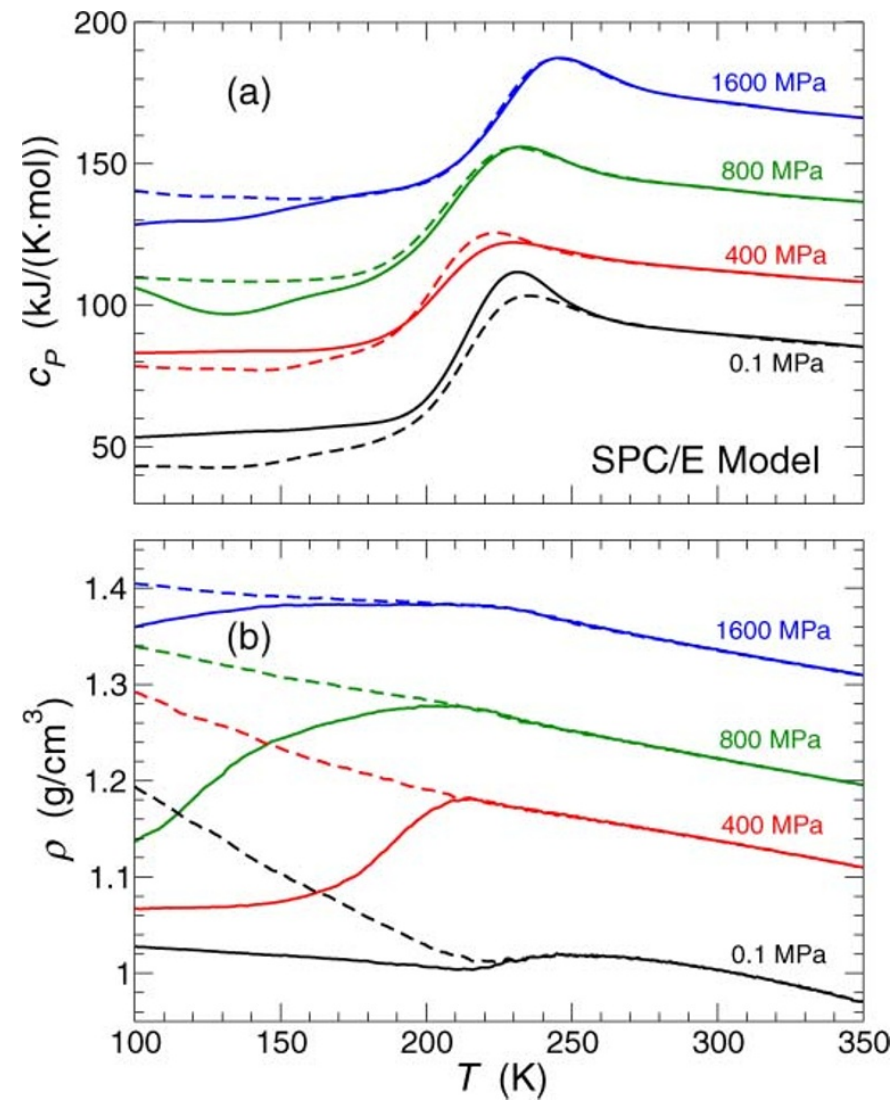

Figure $2 \mid$ Isobaric specific heat and density for glasses of the SPC/E model on heating. (a) Isobaric specific heat, $c_{P}(T)$, and (b) density, $\rho(T)$, upon heating compressed HGW (solid lines) and decompressed HDA (dashed lines) at various pressures. $T_{g}(P)$ (defined by the inflection of $c_{P}(T)$ below its maximum temperature) occurs at nearly the same temperature for compressed HGW and decompressed HDA. However, the behavior of the $c_{P}(T)$ and $\rho(T)$ differ significantly in the glassy state (e.g., at $T<200 \mathrm{~K}$ for all pressures). For clarity, curves in (a) have been successively shifted by $30 \mathrm{~kJ} /(\mathrm{K} \cdot \mathrm{mol})$ for increasing pressure.

function of $P$. Moreover, it can be shown from eq. (2) that a maximum in $D$ corresponds to a minimum in $T_{g}(P)$, and vice versa. Accordingly, the $D_{\max }$-line must intersect the $T_{g}(P)$ locus at the pressure at which $T_{g}(P)$ has a minimum. Additionally, at very low pressure approaching the liquid spinodal, it is known that the SPC/E model exhibits a minimum of $D$, due to "tearing apart" hydrogen bonds at very low density ${ }^{38,49}$. Equation 2 requires that the associated $D_{\text {min }}$-line must intersect with a maximum on $T_{g}(P)$. We do not observe this feature, since $D_{\min }$ occurs at negative pressures that are not explored in this work.

Glass Transition in the Presence of a Liquid-Liquid Phase Transition. We now turn our attention to the ST2 model where the LLPT is accessible in equilibrium simulations and can significantly impact the behavior of glassy states. Due to vast differences in the behavior of $T_{g}(P)$ for various glasses, we examine the $T_{g}(P)$ locus for each glass separately.

$T_{g}$ from Hyperquenching under Pressure. To unambiguously associate a glass with a liquid for a given pressure, the glass should be formed by isobaric quench from the liquid state at that pressure. So far, cooling under pressure is only experimentally feasible for emulsion samples ${ }^{34}$. Fortunately, the experimental complications pose no problem in computational studies. Therefore, we first examine $T_{g}(P)$ obtained by hyperquenching the equilibrium liquid under pressure and reheating at the same $P$ using our standard cooling/heating rate; we refer to the glass transition temperature of the resulting hyperquenched glasses (i.e. HQG) as $T_{g}^{\mathrm{HQG}}(P)$. The behavior of the $T_{g}(P)$ formed in this straight-forward approach provides a useful baseline to interpret the $T_{g}(P)$ behavior we will see for compressed HGW and decompressed HDA.

The $T_{g}^{\mathrm{HQG}}(P)$ locus is shown in Fig. 3a. Qualitatively similar to the $T_{g}(P)$ locus of SPC/E water, we see that $T_{g}^{\mathrm{HQG}}(P)$ for the ST2 model is also negatively sloped at low pressure (due to the diffusion anomaly), and positively sloped at high pressures. The data for the ST2 model does not extend to very high pressures due to crystallization on
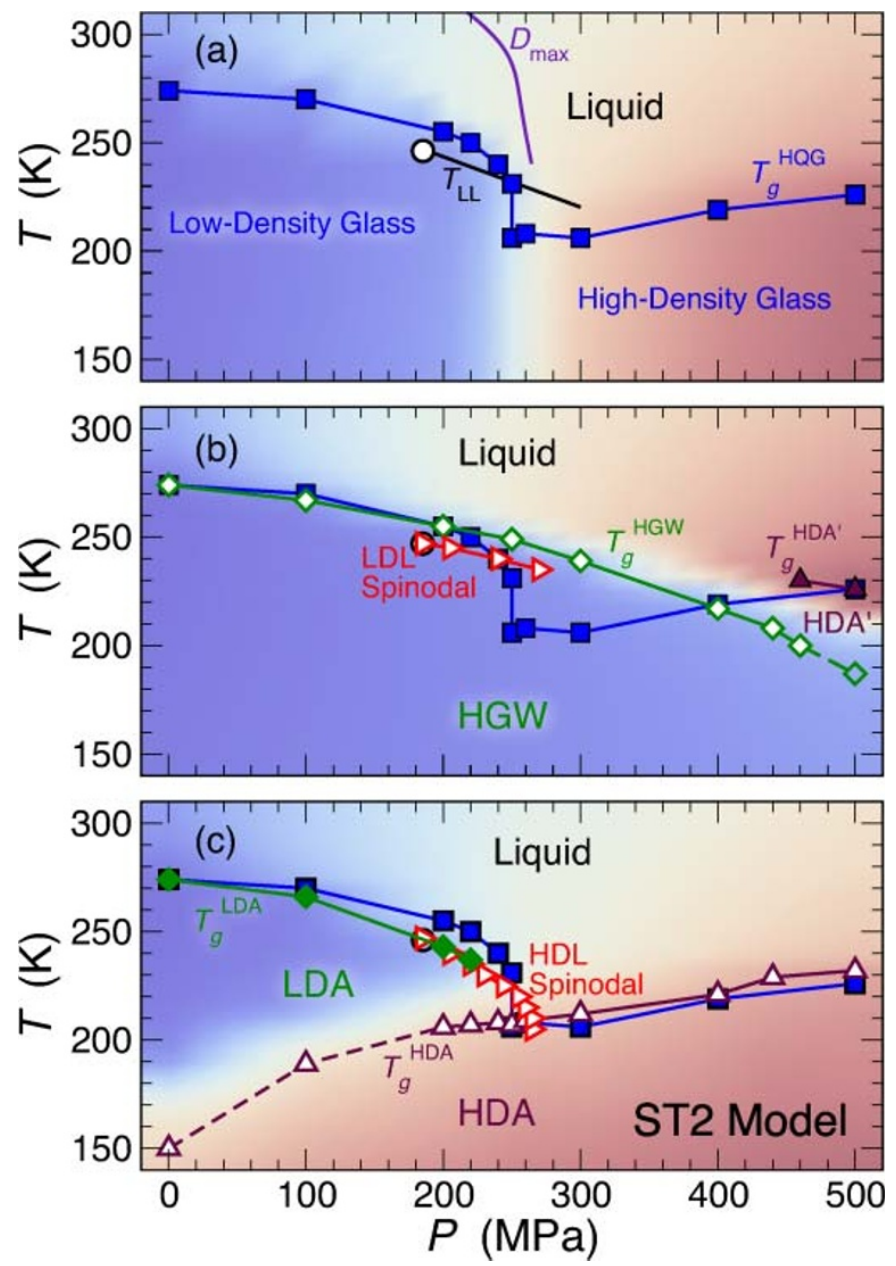

Figure $3 \mid$ Pressure-dependence of the glass transition temperature $T_{g}(P)$ for glasses of the ST2 model. (a) $T_{g}^{\mathrm{HQG}}(P)$ determined by hyperquenching the equilibrium liquid at the desired pressure $P$, and reheating the resulting glass at that same $P$. As discussed in the text, the dramatic change of $T_{g}^{\mathrm{HQG}}(P)$ around $250 \mathrm{MPa}$ can be understood from the location of the equilibrium LLPT line (taken from Ref.13). The $D_{\max }$ locus is taken from Ref.50. The color gradient represents the density $\rho$ of HQG on heating. (b) Comparison of the glass transition temperature of compressed HGW, $T_{g}^{H G W}(P)$, with $T_{g}^{H Q G}(P)$. At low $P, T_{g}^{H G W}(P) \approx T_{g}^{H Q G}(P)$ while at high $P$, compressed HGW converts to a high density glass (HDA') before a glass transition to HDL occurs (violet solid line). The dotted portion of the $T_{g}^{\mathrm{HGW}}(P)$ locus indicates the temperature at which compressed HGW transforms to $\mathrm{HDA}^{\prime}$. Included is the HDL-to-LDL spinodal line from Ref.12. Here the color gradient represents $\rho$ of compressed HGW on heating. (c) Comparison of the glass transition temperature of decompressed HDA, $T_{g}^{\mathrm{HDA}}(P)$, with $T_{g}^{\mathrm{HQG}}(P)$. At high $P$, $T_{g}^{\mathrm{HDA}}(P) \approx T_{g}^{\mathrm{HQG}}(P)$ while at low $P$, decompressed HDA converts to LDA (dashed line) before a glass transition to the liquid occurs at $T_{\mathrm{g}}{ }^{\mathrm{LDA}}(P)$ (green solid line). Here the color gradient is $\rho$ of decompressed HDA on heating, which helps to identify the transformation to LDA. 
heating that complicates determination of $T_{g}$. Interestingly, unlike the SPC/E model case, there is a sharp change in $T_{g}(P)$ at $P=$ $250 \mathrm{MPa}$.

The sharp change of $T_{g}^{\mathrm{HQG}}(P)$ at $P=250 \mathrm{MPa}$ can be understood by superimposing the known location of the LL coexistence line for ST2 (taken from ref.13). Figure 3a shows that, for $P<250 \mathrm{MPa}$, the $T_{g}^{\mathrm{HQG}}(P)$ locus is at higher $T$ than the liquid-liquid coexistence line. Accordingly, on hyperquenching, the liquid falls out of equilibrium (into a low-density glass) before the LLPT occurs, and so $T_{g}^{\mathrm{HQG}}(P)$ is smooth at all $P<250 \mathrm{MPa}$. At $P=250 \mathrm{MPa}$, the $T_{g}^{\mathrm{HQG}}(P)$ locus intersects the LL coexistence line, and consequently the system is now sensitive to the equilibrium LLPT, and so there should be a LLPT before vitrification (into a high-density glass). Accordingly, the large difference in the dynamical properties of LDL (for $P \lesssim 250 \mathrm{MPa}$ ) and HDL (for $P \gtrsim 250 \mathrm{MPa}$ ) is reflected by a large change in $T_{g}^{\mathrm{HQG}}(P)$ at $P=250 \mathrm{MPa}$. A similar discontinuity in $T_{g}(P)$ has been observed in computer simulations of a polyamorphic monatomic liquid ${ }^{33}$.

This dramatic change in the liquid properties is indicated by the presence of two distinctive features in $c_{P}(T)$ upon heating HQG at $P$ $=250 \mathrm{MPa}$ (Fig. 4) (similar characteristics are observed in $\rho(T)$, see
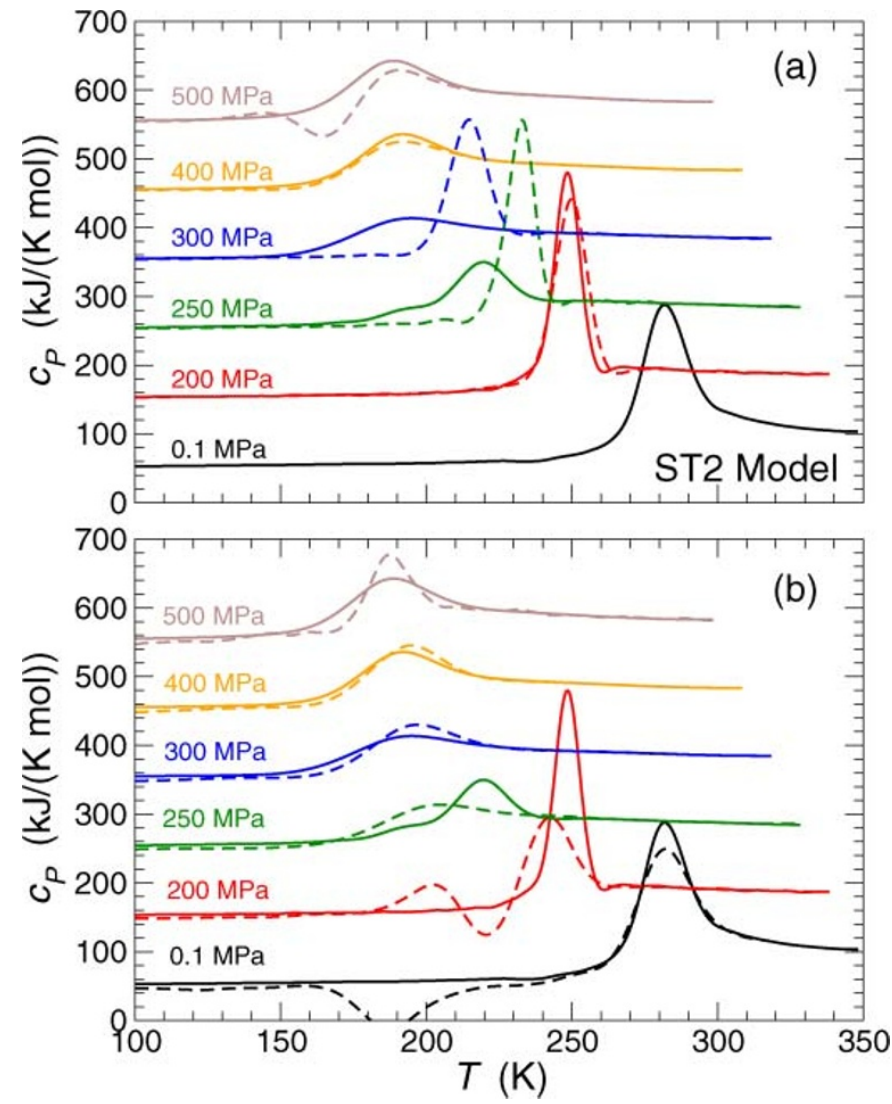

Figure $4 \mid$ Isobaric specific heat for glasses of the ST2 model on heating. Comparison of the isobaric specific heat $c_{P}(T)$ for (a) HQG (solid lines) and compressed HGW (dashed lines) and (b) HQG (solid lines) and decompressed HDA (dashed lines) at various pressures. For clarity of the figure, curves for increasing pressure have been successively shifted vertically by $100 \mathrm{~kJ} /(\mathrm{K} \cdot \mathrm{mol})$ and horizontally by- $10 \mathrm{~K}$. For the case of compressed HGW [panel (a)], there are two features at high pressures. For example, at $P=500 \mathrm{MPa}$, compressed HGW converts to a high density glass similar to HDA at $T \approx 190 \mathrm{~K}$, which on further heating converts to an equilibrium liquid at $T \approx 240 \mathrm{~K}$. Similarly, for decompressed HDA at low pressure [panel (b)], there are two features at low pressures. For example, at $P=0.1 \mathrm{MPa}, \mathrm{HDA}$ converts to LDA (similar to HGW) at $T \approx 150 \mathrm{~K}$, which on further heating converts to an equilibrium liquid at $T=275 \mathrm{~K}$.
Fig. 5). Specifically, in addition to the typical $c_{P}$ peak, characteristic of the glass-to-liquid transition observed at $P<250 \mathrm{MPa}$, a shoulder in $c_{P}(T)$ develops at slightly lower temperature, $T \approx 220 \mathrm{~K}$ (Fig. $4 \mathrm{a}$ ). As we discuss below, this lower- $T$ feature of $c_{P}(T)$ can be associated to the transformation of the high-density glass (HDA) to the low-density glass (LDA); the upper- $T$ feature of $c_{P}(T)$ can be related to a glassto-liquid transition, from the low-density glass to the liquid. At $P=$ $250 \mathrm{MPa}$, this last transformation occurs at the intersection of the $T_{g}^{\mathrm{HQG}}(P)$ and liquid-liquid coexistence $T_{L L}(P)$ loci. At higher pressure, a simpler behavior reemerges. Specifically, for $P>250 \mathrm{MPa}$, hyperquenching the liquid yields a high-density glassy state that connects smoothly with the behavior of the liquid at high pressure (Fig. 3a $)^{43}$.

A minimum in $T_{g}^{\mathrm{HQG}}(P)$ apparently develops at approximately $250 \leq P \leq 300 \mathrm{MPa}$, close to the $\mathrm{P}$ where $T_{g}^{\mathrm{HQG}}(P)$ it exhibits a discontinuity - at the intersection with the $T_{\mathrm{LL}}$ line. This discontinuity is consistent with the fact that, across the $T_{\mathrm{LL}}$ locus, $\tau$ will show a discontinuity with the change in phase. Like the SPC/E model, Fig. 3a also shows that the $D_{\max }$-line approaches the minimum in $T_{g}^{\mathrm{HQG}}(P)$.

$T_{g}$ of Compressed HGW. We next compare the behavior of $T_{g}^{\mathrm{HGW}}(P)$ with that of $T_{g}^{\mathrm{HQG}}(P)$. Since HGW is formed by hyperquenching the liquid at atmospheric pressure, it is natural to expect that, at low pressure, the $T_{g}$ resulting from heating compressed HGW should be very similar to that of the glass formed by hyperquenching the pressurized equilibrium liquid (i.e., HQG). For $P \leq 200 \mathrm{MPa}$, Fig. $3 \mathrm{~b}$ confirms that indeed $T_{g}^{\mathrm{HGW}}(P) \approx T_{g}^{\mathrm{HQG}}(P)$. Moreover, the behavior of $c_{P}(T)$ of the two glasses, even at $\stackrel{g}{T}<T_{g}$, is nearly identical in this pressure range (Fig. 4a). For $P>250 \mathrm{MPa}$, the $T_{g}^{H G W}(P)$ and $T_{g}^{H Q G}(P)$ loci differ significantly. Specifically, while $T_{g}^{\mathrm{HQG}}(P)$ is
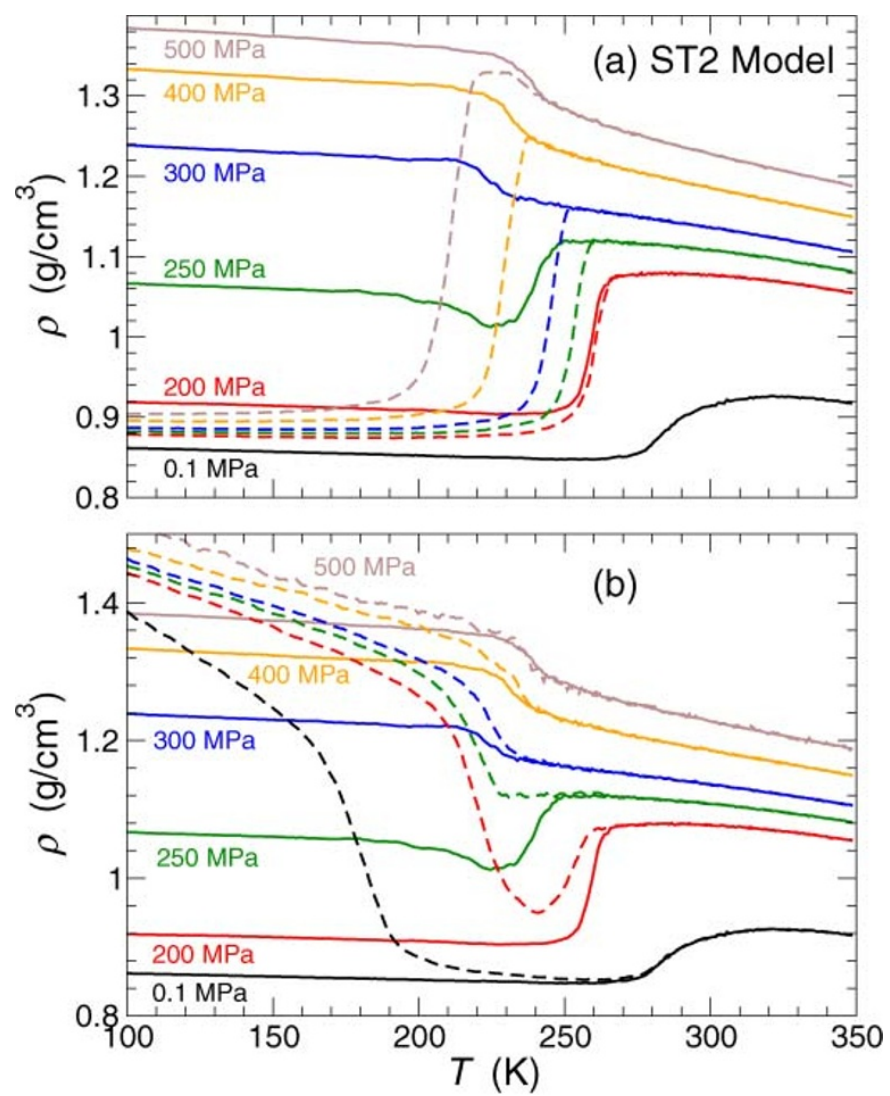

Figure $5 \mid$ Density for glasses of the ST2 model on heating. Comparison of the density $\rho(T)$ for (a) HQG (solid lines) and compressed HGW (dashed lines), and for (b) HQG (solid lines) and decompressed HDA (dashed lines) at various pressures. 
affected by the proximity to the LLPT, $T_{g}^{\mathrm{HGW}}(P)$ is nearly insensitive to the presence of the liquid-liquid transition.

We can understand the difference between the $T_{g}^{H G W}(P)$ and $T_{g}^{H Q G}(P)$ loci at high pressure based on the preparation of compressed HGW. Since we compress HGW at very low $T$, compressed HGW cannot relax during compression; thus, compressed HGW retains the characteristics of the low-density glass state, even at pressures where the high-density state may have lower free energy. This is analogous to the fact that HDA can be decompressed to atmospheric pressure and retain its high density, provided the temperature is kept sufficiently low. Since the equilibrium LLPT occurs at temperatures below $T_{g}^{\mathrm{HGW}}(P)$ (for the present heating/cooling rate), the $T_{g}^{\mathrm{HGW}}(P)$ locus continues as a smooth extension of its low pressure behavior to $P$ much larger than $250 \mathrm{MPa}$ (where the $T_{g}(P)$ of HQG has an apparent discontinuity).

Since compressed HGW remains mechanically stable for $P>P_{c}$ $\left(P_{c}=190 \mathrm{MPa}\right)$, it is natural to ask to what liquid compressed HGW transforms on heating at high pressures. We separately consider the cases $P_{c}<P<400 \mathrm{MPa}$ and $P>400 \mathrm{MPa}$, since at $P \approx 400 \mathrm{MPa}$ the $T_{g}^{\mathrm{HGW}}(P)$ and $T_{g}^{\mathrm{HQG}}(P)$ loci intersect one another.

In the range $P_{c}<P<400 \mathrm{MPa}$, compressed HGW transforms to HDL without intermediate phases. That the final liquid is HDL can be understood by noting the location of the LDL-to-HDL spinodal (see Fig. 3b). It can be seen that $T_{g}^{\mathrm{HGW}}(P)$ is greater than the spinodal temperature. Therefore, on heating, the glass transition must be from HGW to HDL, since HDL is the only (meta)stable liquid at these $(P, T)$. This is confirmed by the dramatic increase in density at $T_{g}^{\mathrm{HGW}}(P)$ shown in Fig. 5a for $P_{c}<P \leq 400 \mathrm{MPa}$.

At $P>400 \mathrm{MPa}$, the final liquid resulting from heating compressed $\mathrm{HGW}$ is also HDL. However, the progression to the HDL state is more complex at these pressures. At $P>400 \mathrm{MPa}, T_{g}^{\mathrm{HGW}}(P) \leq T_{g}^{\mathrm{HQG}}(P)$ and, as a result, when compressed HGW reaches $T_{g}$, no metastable liquid state is accessible. Thus, HGW makes a glass-glass transition to an HDA-like glass - the glassy extension of HDL. We refer to this form as $\mathrm{HDA}^{\prime}$ since it has similar (but not identical) properties to $\mathrm{HDA}$ on decompression. The $\mathrm{HGW} \rightarrow \mathrm{HDA}^{\prime}$ transformation is dominated by an orientational reordering of the molecules, and does not require significant translational displacements. For $P \gtrsim 450 \mathrm{MPa}$ - the glass-glass and glass-liquid transitions are well separated, and we can detect two distinct transformations in $c_{P}(T)$ (Fig. $4 \mathrm{a}$ ) - the first being $\mathrm{HGW} \rightarrow \mathrm{HDA}^{\prime}$, followed on heating by $\mathrm{HDA} \rightarrow$ liquid, defining $T_{g}^{\mathrm{HDA}^{\prime}}(P)$ which roughly coincides with $T_{g}^{\mathrm{HQG}}(P)$. It is important to notice that the glass-glass $\mathrm{HGW} \rightarrow \mathrm{HDA}^{\prime}$ transition is a natural continuation of the $T_{g}^{\mathrm{HGW}}(P)$ from lower $P$, showing that the shape of the $T_{g}^{\mathrm{HGW}}(P)$ depends only on the form of the glass, and not the liquid (or glass) to which HGW transforms. Hence, the HGW-to-HDA' transition is a result of HGW reaching its kinetic (not thermodynamic) limit of stability, and becoming caught in another out-of-equilibrium state, HDA', before the liquid becomes available upon further heating. Considering that LDA and HGW are largely the same, it follows that the LDA-to-HDA' transition upon heating at high pressures is not related to the LDL-to-HDL spinodal, but instead to the LDA $T_{g}$. A similar scenario holds for the case of decompressed HDA, as discussed in the next section.

$T_{g}$ of Decompressed HDA. We finally examine the $T_{g}(P)$ locus of decompressed HDA. This form of HDA is most closely related to that of experimentally prepared $\mathrm{uHDA}^{51}$. The behavior of $T_{g}^{\mathrm{HDA}}(P)$ is easiest to understand at high pressure since HDA is formed under high pressure conditions. Specifically, we expect that the high pressure behavior of $T_{g}^{\mathrm{HDA}}(P)$ should approximately follow that of the glass from the hyperquenched liquid at the same pressure. Indeed, Fig. $3 \mathrm{c}$ shows that, for $P \gtrsim 250 \mathrm{MPa}, T_{g}^{\mathrm{HDA}}(P) \approx T_{g}^{\mathrm{HQG}}(P)$. We note that at $P \approx 260 \mathrm{MPa}$, the $T_{g}^{H D A}(P)$ locus intersects the HDL-to-LDL spinodal on decompression. Therefore, the liquid to which HDA transforms at $P \gtrsim 260 \mathrm{MPa}$ is HDL.
We next consider the behavior of $T_{g}^{H D A}(P)$ at $P<250 \mathrm{MPa}$, where the equilibrium liquid forms a low-density glass on isobaric quenching. Just as in experiments, HDA in our simulations is mechanically stable at liquid $\mathrm{N}_{2}$ temperatures down to atmospheric pressure. Furthermore, as known experimentally, but not observed for the SPC/E model, decompressed HDA shows a clear conversion to a low density amorphous glass upon heating, normally referred to as LDA. The conversion to LDA, as well as the conversion of LDA to a liquid upon further heating, appear as two distinct features in the specific heat (Fig. 4b). This is particularly clear at $P=0.1$ and $200 \mathrm{MPa}$ where $c_{P}(T)$ exhibits minima at $T \approx 190$ and $230 \mathrm{~K}$ (respectively) below the temperature at which the $c_{P}$ maximum, that is characteristic of the glass transition, occurs.

As observed in experiments, the HDA-to-LDA (glass-glass) transformation, readily seen by a similar exothermic feature (Fig. $4 \mathrm{~b}$ ), is accompanied by a dramatic decrease of density (Fig. 5b). Like the conversion of compressed HGW to HDA' at high pressure discussed in the previous subsection, the HDA-to-LDA transformation is primarily associated with a reorientation of the molecules without significant translational motion. Figure $3 \mathrm{c}$ shows that the locus of the HDA-to-LDA (dashed violet line) connects smoothly with the $T_{g}^{\mathrm{HDA}}(P)$ locus (solid violet line) at high pressure. This demonstrates that the HDA-to-LDA transition is a result of HDA reaching its $T_{g}$; rather than transforming to the liquid at $T_{g}$, the system becomes caught in another out-of-equilibrium state, LDA, before the liquid becomes available upon further heating. It follows that the HDA-toLDA transition is not related to the thermodynamic HDL-to-LDL spinodal. However, the HDL-to-LDL spinodal on decompression does signal the terminal pressure of the HDA-to-LDA conversion locus. At large pressures, $P \gtrsim 250 \mathrm{MPa}$, HDA converts directly to HDL.

Experimentally, LDA is known to have similar $T_{g}$ to $\mathrm{HGW}$ at atmospheric pressure ${ }^{52}$. Therefore, we expect that the $T_{g}(P)$ locus from heating LDA should be similar to both $T_{g}(P)$ for compressed HGW and HQG. Indeed, Figs. $3 \mathrm{~b}$ and $3 \mathrm{c}$ show that for $P<100 \mathrm{MPa}$, $T_{g}^{\mathrm{LDA}}(P) \approx T_{g}^{\mathrm{HGW}}(P) \approx T_{g}^{\mathrm{HQG}}(P)$. Nearing the region of the LLPT, the behavior of the $T_{g}$ loci for the different low-density glasses have similar pressure dependence but no longer coincide - not surprising since the density of LDA is noticeably different from compressed $\mathrm{HGW}$ at $P \approx 200 \mathrm{MPa}$ and $T<260 \mathrm{~K}$ (Fig. 5b).

Relationship to Experiments. The results obtained for the SPC/E (without a LLPT) and the ST2 (with a LLPT) models present clearly distinguishable scenarios for the behavior of the $T_{g}(P)$ loci of the different glasses. In this section, we compare available experimental data for real water with these scenarios. In short, we find that the available experimental data for (i) the $T_{g}(P)$ loci of LDA and HDA, (ii) the pressure-dependence of the LDA-to-HDA and HDA-to-LDA transition temperatures, and (iii) the location of the $D_{\text {max }}$-line for liquid water are consistent with the LLPT scenario of the ST2 model, and inconsistent with the simulations using the SPC/ E model that lack an accessible LLPT. We explain the comparisons in the following. To simplify the discussion, we use $T_{g}(P)$ to refer to both the locus of glass-glass and glass-liquid transitions.

We first consider the ST2 model scenario, where HDA and LDA (or HGW) exhibit distinct $T_{g}(P)$ loci. Experimental data for the transitions of the amorphous solids are shown in Fig. 6, which can be compared with Fig. 3. We observe several key qualitative similarities. Specifically, we see that (i) the experimental $T_{g}(P)$ locus of HDA on heating ${ }^{35}$ and the decompression induced HDA-to-LDA transition line ${ }^{26}$ are both positively sloped and can be identified with the qualitative behavior of the $T_{g}^{\mathrm{HDA}}$ locus of the ST2 model; (ii) conversely, the compression induced LDA-to-HDA transition line ${ }^{26}$ is negatively sloped, and, if we associate this line with a pressure induced glass-glass transition, the behavior is consistent with the ST2 model; (iii) the location of the $D_{\max }$ line is consistent with a 
change of the $T_{g}(P)$ locus from one like LDA at low pressure (negative slope), to one like HDA (positive slope) at high pressure; (iv) the calorimetric $T_{g}$ of LDA is higher than the calorimetric $T_{g}$ of HDA at ambient pressure ${ }^{35}$. Based on the similarity between the ST2 results and experiments, we can make a crude prediction for the experimental $T_{g}(P)$ locus of LDA (or HGW) on heating (Fig. 6). The negative slope of the predicted $T_{g}(P)$ locus is supported by the fact that $D$ has anomalous pressure dependence in this region. Confirmation of such qualitative behavior would strongly support the possibility of a LLPT.

While the similarities are favorable for the scenario of the ST2 model, there are complicating factors based on differences with the experimental details that we should consider. The first complication regards possible differences between the behavior of eHDA and uHDA. The experimental $T_{g}(P)$ locus $^{35}$ for HDA (Fig. 6) is derived from eHDA (annealed at low pressure), while our simulations examine uHDA. $T_{g}$ for these two forms is very similar. However, they apparently differ in the nature of the transformation: careful annealing just above $T_{g}$ for eHDA results in an apparently high-density liquid state for $P \leq 300 \mathrm{MPa}^{35}$, while uHDA transforms immediately to LDA on heating at low pressure ${ }^{53}$ (as found here for the ST2 model). Fig. 6 includes the compression-induced LDA-to-HDA and decompression-induced HDA-to-LDA transition line from ref.26. These experiments were performed using uHDA. The slope of the experimental $T_{g}^{\mathrm{HDA}}(P)$ locus $^{35}$ is very similar to the slope of the experimental HDA-to-LDA transition line ${ }^{26}$ (see Fig. 6). This is consistent with the conclusion that the HDA-to-LDA transition is a result of HDA reaching its $T_{g}$, rather than being associated with the HDL-to-LDL spinodal. The experimental difference between eHDA and uHDA seems to be that the HDA-to-LDA transition is shifted to higher temperature in the former ${ }^{29}$. This difference opens a window where HDL is accessible in isobaric heating experiments done with $\mathrm{eHDA}^{35}$, whereas UHDA transforms to LDA without access to HDL. Another complication is that (above atmospheric

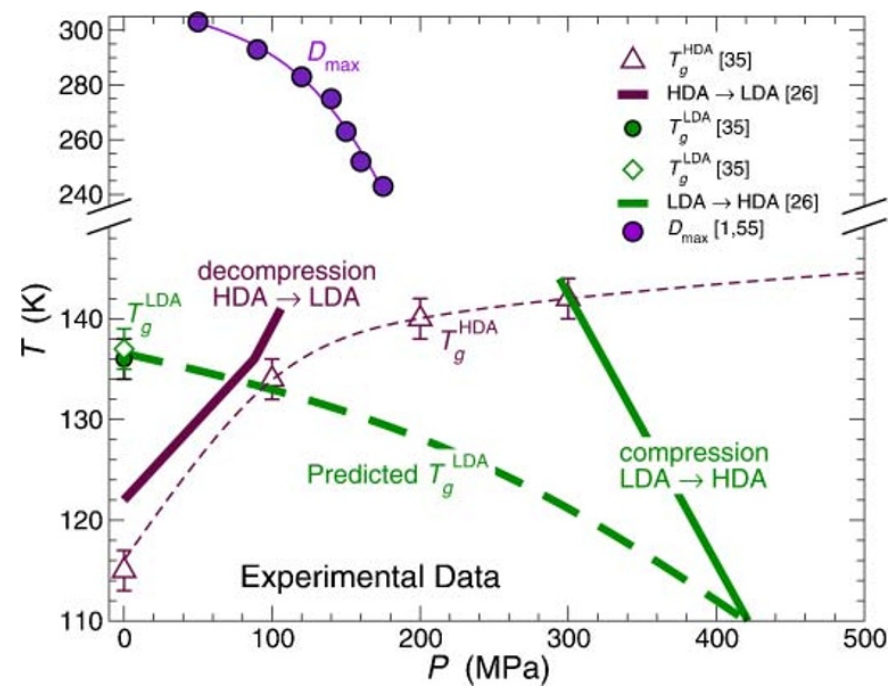

Figure 6 The experimental behavior of $T_{g}(P)$ for LDA and HDA. Data for $T_{g}$ are primarily from ref. ${ }^{35}$. We also compare with data for the isothermal, pressure driven LDA/HDA transition ${ }^{26}$. The difference in path and rate between these experiments precludes a quantitative comparison. However, the data show the same qualitative features as the ST2 model that exhibits a LLPT (Fig. 3). Given these facts, the dashed green line offers a prediction for the approximate behavior of $T_{g}(P)$ for LDA. The prediction is determined by scaling and shifting $T_{g}^{\mathrm{HDA}}$ from the ST2 model to match the experimental $T_{g}^{\mathrm{HDA}}$ (the resulting locus is indicated by the dashed violet line); this scaling factor is then applied to $T_{g}^{\mathrm{HGW}}$ from the ST2 model, and shifted so that it matches experiments at atmospheric pressure. pressure) the experimental $T_{g}^{\mathrm{HDA} 35}$ has been obtained volumetrically. However, simulations indicate that $T_{g}$ measured from calorimetry and volume experiments are very close to each other ${ }^{54}$; we made a similar analysis using our volume data, and find consistent results with the calorimetric method (see supplementary information).

We next consider a comparison of experimental data with the SPC/E scenario. In addition to the glass transition temperatures of LDA and HDA, Fig. 6 also shows the location of the experimental $D_{\max }$ line ${ }^{1,55}$. To show that the experimental data is inconsistent with the SPC/E scenario, we note that in the SPC/E scenario: (i) there is a single $T_{g}(P)$ locus for all amorphous ices, and (ii) the $T_{g}(P)$ locus exhibits a single minimum that intersects the $D_{\max }$-line. Figure 6 indicates that points (i) and (ii) cannot be simultaneously satisfied. Specifically, if there were a single $T_{g}(P)$ locus, then its minimum must occur at approximately $P \leq 115 \mathrm{MPa}$ in order to intersect the LDA and HDA $T_{g}$ loci in Fig. 6 . However, the pressure of the $D_{\max }$-line, which should coincide with a minimum of $T_{g}(P)$ locus, clearly extrapolates to much larger pressure, $P \geq 190 \mathrm{MPa}$. Hence, conditions (i) and (ii) cannot both be satisfied, and so the SPC/E picture is not supported by the experiments. In other words, observation of two distinct values of $T_{g}(\mathrm{LDA})$ and $T_{g}(\mathrm{HDA})$ at approximately $200 \mathrm{MPa}$, where the Dmax line intersects, can be used as a criterion to positvely identify a liquid-liquid phase transition scenario and to rule out a scenario without such a transition. The predictions made here combined with the experimental scenario strongly suggest this to be the case, but definite experimental confirmation remains an open issue.

\section{Discussion}

We have evaluated the pressure dependence of $T_{g}$ for various forms of amorphous water in two simulation models, one with an accessible liquid-liquid transition, and one without. Our findings indicate a much richer behavior for $T_{g}(P)$ in the presence of a LLPT, and that this behavior appears to be consistent with the available experimental data. The behavior of $T_{g}$ from simulations in the absence of an LLPT is inconsistent with the available experimental data. Hence our findings support the possibility of multiple liquid states and a critical point in supercooled water. Based on these results, we have made a rough prediction for $T_{g}(P)$ of LDA. Further experimental work to evaluate the $T_{g}(P)$ loci of HGW or LDA, will be valuable to confirm or refute these hypotheses.

\section{Methods}

Simulation models and methods. Our findings are based on extensive molecular dynamics (MD) simulations of water using two different models: (i) the extended simple point charge (SPC/E) model ${ }^{56}$, and (ii) the Stillinger "ST2" potential ${ }^{57}$. These models have been extensively applied to the study of thermodynamic and dynamic properties of supercooled water and, to a lesser degree, to the study of glassy water ${ }^{28}$ Both models reproduce qualitatively the most common anomalies characteristic of liquid water. The primary difference between the models, and the reason we select them, is that the ST2 model exhibits a LLPT that is readily accessible by computer simulation $^{4,11,12,17}$, while the SPC/E model does not. If it exhibits a LLPT, it occurs at a temperature well below the lowest temperatures accessible in equilibrium simulations $^{37-39}$.

The basic potential parameters and simulation protocols, such as simulation time step and cutoff distance used for the truncation of the interactions, etc. are described in previous studies: we follow precisely the parameterization described in refs.38 and 12 for the SPC/E and ST2 models, respectively. The temperature and pressure are controlled using the Berendsen technique ${ }^{58}$. All data are averaged over 20 independent samples to improve statistics. For glasses obtained from an initially liquid state, we use a 1000-molecule cubic system with periodic boundary conditions. For glasses obtained from the amorphization of ice $\mathrm{I}_{h}$, we use a system of 1024 molecules in a rectangular unit cell (with periodic boundary conditions) that matches the unit cell geometry of ice $\mathrm{I}_{h}$. Proton disorder in the initial ice configuration is generated by identifying closed hydrogen bond loops, and exchanging hydrogens between molecules, as described in Ref.59. For the ST2 model, some samples crystallize on heating the glass for $P \gtrsim 300 \mathrm{MPa}$. We have excluded these samples from our analysis.

Preparation of Glassy States. The glass obtained by rapidly quenching bulk water at ambient pressure is referred to as hyperquenched glassy water $(\mathrm{HGW})^{31}$. To obtain $\mathrm{HGW}$ in simulation, we first equilibrate liquid water at relatively high temperature $(T$ $=300 \mathrm{~K}$ for SPC/E, and $T=350 \mathrm{~K}$ for ST2) and $P=0.1 \mathrm{MPa}$. We isobarically cool the liquid to $T=80 \mathrm{~K}$ at a rate $q_{T}=30 \mathrm{~K} / \mathrm{ns}$. To study the pressure dependence of $T_{g}$ 

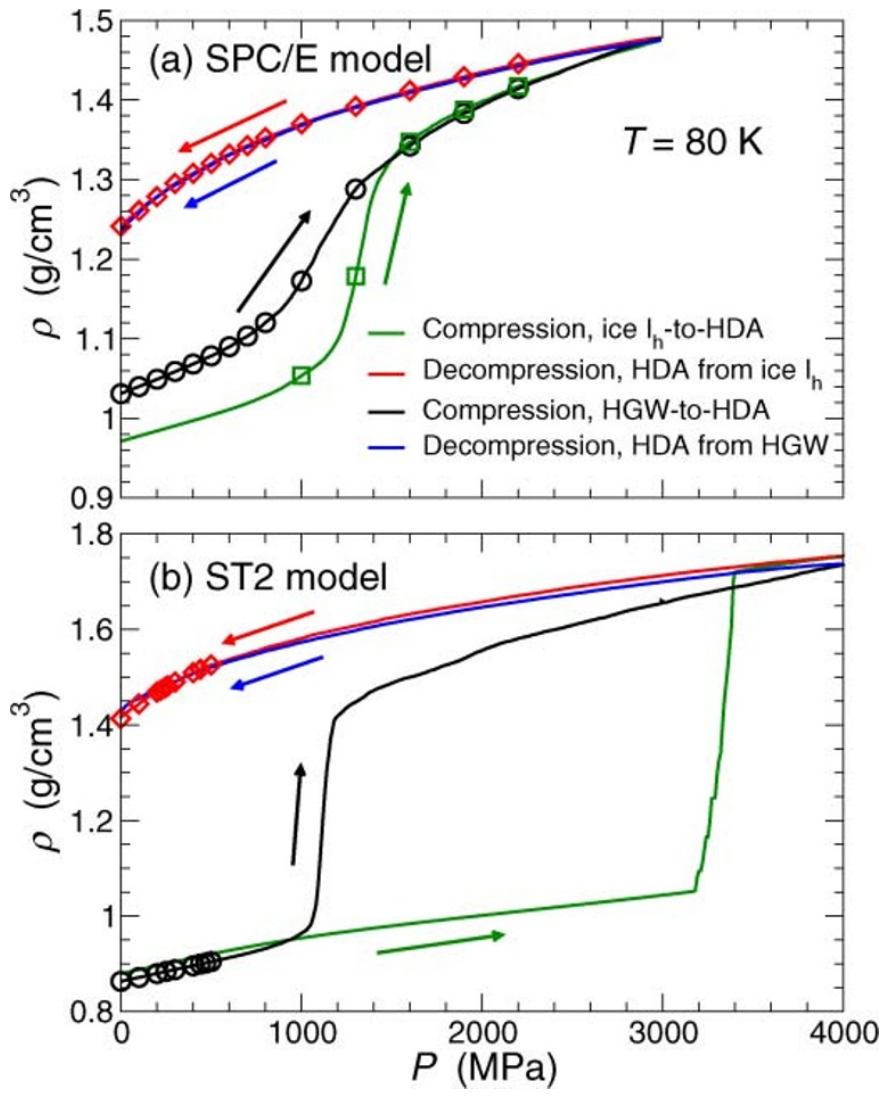

Figure 7 | Compression/Decompression behavior of LDA, HDA, and ice $\mathrm{I}_{\boldsymbol{h}}$. Evolution of density at $T=80 \mathrm{~K}$ upon compressing ice $\mathrm{I}_{h}$ (green line) and HGW (black line) to form HDA, followed by decompression of HDA (red and blue lines correspond to decompression of HDA obtained from ice $\mathrm{I}_{h}$ and HGW, respectively). Results are for (a) the SPC/E and (b) the ST2 model. In both models, the sudden changes in density correspond to the ice $\mathrm{I}_{h}$-to-HDA (green line) or HGW-to-HDA (black line) transition, respectively. Symbols indicate the starting densities for glass configurations that are heated at fixed $P$ to determine $T_{g}$, shown in subsequent figures.

for the case of HGW, we first isothermally compress HGW at a rate $q_{P}=300 \mathrm{MPa} / \mathrm{ns}$ from $P=0.1 \mathrm{MPa}$ to the desired $P$ at $T=80 \mathrm{~K}$; we then isobarically heat the sample at the same $P$ and recover a pressurized liquid state. We note that $\mathrm{HGW}$ can be compressed at $T=80 \mathrm{~K}$ to $P \approx 700 \mathrm{MPa}$, for the SPC/E model case, and to $P \approx$ $1000 \mathrm{MPa}$, for the ST2 model case; further compression causes HGW to collapse to an HDA-type glass for both models (Fig. 7). A similar collapse from an LDA-type glass to an HDA-type glass is observed experimentally ${ }^{26,60}$.

We also obtain HDA by following the corresponding experimental procedure ${ }^{46}$, i.e., we isothermally compress proton-disordered ice $\mathrm{I}_{h}$ (at the rate $q_{P}$ ) at $T=80 \mathrm{~K}$ to high pressure. Since we do not anneal HDA, the closest experimental counterpart to our procedure is "unrelaxed" HDA (uHDA), as opposed to relaxed (rHDA) or expanded (eHDA) treatments ${ }^{51}$. Figure 7 shows the density as function of pressure during compression of ice $\mathrm{I}_{h}$; the results differ significantly between the two models. For the SPC/E model, the collapse to HDA happens over a narrow, but gradual and continuous range of pressure, $1100 \lesssim P \lesssim 1500 \mathrm{MPa}$, while for the ST2 model, the collapse happens over a very narrow window around $P=3200 \mathrm{MPa}$. Given that these results are an average over independent compressions, examining individual runs shows that the collapse of ice $\mathrm{I}_{h}$ is indeed discontinuous in the case of the ST2 model; the width in the average density change in Fig. $7 \mathrm{a}$ is a result of the fact that different realizations collapse at slightly different $P$. An examination of individual runs for SPC/E water shows that the behavior of each realization has the same gradual behavior as the average. The difference in the sharpness in the two models is presumably a consequence of the fact that ST2 water has a clear LLPT and LLCP, accessible to equilibrium simulations, while SPC/E water does not (at least for our rapid cooling and compression rates). We note that the experimental amorphization of ice $\mathrm{I}_{h}{ }^{46}$ more closely resembles the behavior of ST2 water, albeit at significantly smaller $P$. The ST2 model shows a larger transition pressure, both due to the rapid pressurization in the simulation and because ST2 so strongly emphasizes tetrahedral (ice-like) structure. We note that for both water models and also in experiments ${ }^{28}$, the conversion of ice $\mathrm{I}_{h}$ to HDA occurs at higher pressures than the pressures at which
HGW converts to HDA. However, the structure of HDA obtained by compression of HGW or ice $\mathrm{I}_{h}$ is indistinguishable. As a result, decompression (at rate $q_{P}$ ) of HDA formed from ice or HGW shows the same $P(\rho)$ dependence (Fig. 7).

To obtain LDA we follow the typical experimental procedure ${ }^{24}$, i.e., we first decompress HDA to low pressures (at rate $q_{P}$ ) at $T=80 \mathrm{~K}$ and then, heat decompressed HDA at constant pressure (at rate $q_{T}$ ). As in the experimental case, HDA does not convert to LDA at $P=0.1 \mathrm{MPa}$ at low $T$. Conversion to LDA only occurs on heating decompressed-HDA. The starting HDA configurations are obtained by isothermal compression of ice $\mathrm{I}_{h}$ at $T=80 \mathrm{~K}$, as previously explained. The main text shows that the HDA-to-LDA transformation can be clearly identified for the ST2 case; for SPC/E, the HDA-to-LDA transformation is more gradual, and not easily delineated $^{44}$. We note that for both the ST2 and SPC/E potentials, the decompressed HDA prepared from an initial compression of ice or HGW for each model is nearly indistinguishable in terms of density (see Fig. 7) and structure (as measured by pair correlations).

Determination of $T_{g}$. Experimentally, $T_{g}$ is typically determined from differential scanning calorimetry experiments where the sample is heated at a fixed rate at ambient pressure ${ }^{30,32}$. The glass transition is apparent from a peak in the specific heat $c_{P}(T)$, the height and width of which depend on fragility ${ }^{48}$. The specific value of $T_{g}$ can be assigned by a variety of methods; we assign $T_{g}$ as the temperature corresponding to the inflection point of $c_{P}(T)$ on heating. More specifically, we evaluate the (nonequilibrium) isobaric specific heat, $c_{P}(T)=(\partial H / \partial T)_{P}$, by heating glasses at the same rate $q_{T}=30 \mathrm{~K} / \mathrm{ns}$ used to cool initial samples. To calculate $c_{P}(T)$, we calculate the energy $U(T)$ and volume $V(T)$ of the system as function of $T$, the enthalpy being $H(T)$ $=U(T)+P V(T)$. We associate $T_{g}(P)$ with the inflection point of $c_{P}(T)$ prior to the glass transition maximum. Thus, $T_{g}(P)$ can be obtained from the location of the maximum in $d c_{P}(T) / d T$ that occurs at temperature below the $c_{P}(T)$ glass transition maximum.

We have tested several common definitions for $T_{g}$, for example by the intersection of a linear extrapolation of the low $T$ glass behavior of $c_{P}(T)$ with its linear rising part at higher $T$, before the $c_{P}(T)$ maximum occurs. This is usually referred as the "onset" glass transition temperature, $T_{g \text {,onset }}$. While our $T_{g}$ values are slightly higher than the corresponding $T_{g, \text { onset }}$ values, the qualitative pressure dependence of $T_{g}$ we report is robust for any reasonable definition of $T_{g}$. Note that DSC methods typically do not operate at low $T$ and high $P$ at the same time; consequently, there is no experimental standard method to measure $T_{g}$ at large pressure. In these cases, $T_{g}$ can be measured from dilatometry experiments (see SI).

1. Angell, C. A. In Franks, F. (ed.) Water: A Comprehensive Treatise vol. 7, 1-81 (Plenum, New York, 1982).

2. Mishima, O. \& Stanley, H. E. The relationship between liquid, supercooled and glassy water. Nature 396, 329 (1998).

3. Debenedetti, P. G. Supercooled and glassy water. J. Phys.: Condens. Matter 15, R1669-R1726 (2003).

4. Poole, P. H., Sciortino, F., Essmann, U. \& Stanley, H. Phase behaviour of metastable water. Nature 360, 324-328 (1992).

5. Mishima, O. \& Stanley, H. E. Decompression-induced melting of ice iv and the liquid-liquid transition in water. Nature 392, 192 (1998).

6. Speedy, R. J. Stability-limit conjecture: An interpretation of the properties of water. J. Phys. Chem. 86, 982-991 (1982).

7. Sastry, S., Debenedetti, P., Sciortino, F. \& Stanley, H. E. Singularity-free interpretation of the thermodynamics of supercooled water. Phys. Rev. E 53, 6144-6154 (1996).

8. Poole, P. H., Sciortino, F., Essmann, U. \& Stanley, H. E. Spinodal of liquid water Phys. Rev. E 48, 3799 (1993).

9. Tanaka, H. A self-consistent phase diagram for supercooled water. Nature $\mathbf{3 8 0}$ 328-330 (1996)

10. Sciortino, F., Poole, P. H., Essmann, U. \& Stanley, H. E. Line of compressibility maxima in the phase diagram of supercooled water. Phys. Rev. E 55, 727-737 (1997).

11. Harrington, S., Zhang, R., Poole, P. H., Sciortino, F. \& Stanley, H. Liquid-liquid phase transition: Evidence from simulations. Phys. Rev. Lett. 78, 2409-2412 (1997).

12. Poole, P. H., Saika-Voivod, I. \& Sciortino, F. Density minimum and liquid-liquid phase transition. J. Phys.: Condens. Matter 17, L431-L437 (2005).

13. Cuthbertson, M. J. \& Poole, P. H. Mixturelike behavior near a liquid-liquid phase transition in simulations of supercooled water. Phys. Rev. Lett. 106, 115706 (2011).

14. Yamada, M., Mossa, S., Stanley, H. E. \& Sciortino, F. Interplay between timetemperature-transformation and the liquid-liquid phase transition in water. Phys Rev. Lett. 88, 195701 (2002).

15. Brovchenko, I., Geiger, A. \& Oleinikova, A. Multiple liquid-liquid transitions in supercooled water. J. Chem. Phys. 118, 9473 (2003).

16. Paschek, D. How the liquid-liquid transition affects hydrophobic hydration in deeply supercooled water. Phys. Rev. Lett. 94, 217802 (2005).

17. Liu, Y., Panagiotopoulos, A. Z. \& Debenedetti, P. G. Low-temperature fluid-phase behavior of ST2 water. J. Chem. Phys. 131, 104508 (2009).

18. Sastry, S. \& Angell, C. A. Liquid-liquid phase transition in supercooled silicon. Nat. Materials 2, 739-43 (2003). 
19. Vasisht, V. V., Saw, S. \& Sastry, S. Liquid-liquid critical point in supercooled silicon. Nat. Phys. 7, 549 (2011).

20. Hsu, C. W., Largo, J., Sciortino, F. \& Starr, F. W. Hierarchies of networked phases induced by multiple liquid-liquid critical points. Proc. Natl. Acad. Sci. USA 105, 13711-13715 (2008).

21. Dai, W., Hsu, C. W., Sciortino, F. \& Starr, F. W. Valency dependence of polymorphism and polyamorphism in dna-functionalized nanoparticles. Langmuir 26, 3601-3608 (2010).

22. Zhang, Y. et al. Density hysteresis of heavy water confined in a nanoporous silica matrix. Proc. Natl. Acad. Sci. USA 108, 12206-12211 (2011).

23. Mancinelli, R., Bruni, F. \& Ricci, M. A. Controversial evidence on the point of minimum density in deeply supercooled confined water. J. Phys. Chem. Lett. 1 1277-1282 (2010)

24. Mishima, O., Calvert, L. D. \& Whalley, E. An apparently first-order transition between two amorphous phases of ice induced by pressure. Nature 314, 76 (1985).

25. Mishima, O., Takemura, K. \& Aoki, K. Visual observations of the amorphous amorphous transition in $\mathrm{H}_{2} \mathrm{O}$ under pressure. Science 254, 406-408 (1991).

26. Mishima, $\mathrm{O}$. Reversible first-order transition between two $\mathrm{H}_{2} \mathrm{O}$ amorphs at $\sim 0.2 \mathrm{GPa}$ and $\sim 125$ K. J. Chem. Phys. 100, 5910-5912 (1994).

27. Winkel, K., Elsaesser, M. S., Mayer, E. \& Loerting, T. Water polyamorphism: reversibility and (dis)continuity. J. Chem. Phys. 128, 044510 (2008).

28. Loerting, T. \& Giovambattista, N. Amorphous ices: experiments and numerical simulations. J. Phys.: Condens. Matter 18, R919-R977 (2006).

29. Winkel, K., Mayer, E. \& Loerting, T. Equilibrated high-density amorphous ice and its first-order transition to the low-density form. J. Phys. Chem. B 115, 1414114148 (2011).

30. Johari, G. P., Hallbrucker, A. \& Mayer, E. The glass-liquid transition of hyperquenched water. Nature 330, 552-553 (1987).

31. Brüggeller, P. \& Mayer, E. Complete vitrification in pure liquid water and dilute aqueous solutions. Nature 288, 569-571 (1980).

32. Kohl, I., Bachmann, L., Hallbrucker, A., Mayer, E. \& Loerting, T. Liquid-like relaxation in hyperquenched water at $\leq 140 \mathrm{~K}$. Phys. Chem. Chem. Phys. 7, 32103220 (2005).

33. Xu, L., Buldyrev, S. V., Giovambattista, N., Angell, C. A. \& Stanley, H. E. A monatomic system with a liquid-liquid critical point and two distinct glassy states. J. Chem. Phys. 130, 054505 (2009).

34. Mishima, O. \& Suzuki, Y. Vitrification of emulsified liquid water under pressure. J. Chem. Phys. 115, 4199-4202 (2001).

35. Seidl, M. et al. Volumetric study consistent with a glass-to-liquid transition in amorphous ices under pressure. Phys. Rev. B 83, 100201 (2011).

36. Andersson, O. Glass-liquid transition of water at high pressure. Proc. Natl. Acad. Sci. USA 108, 11013-11016 (2011).

37. Harrington, S., Poole, P. H., Sciortino, F. \& Stanley, H. E. Equation of state of supercooled water simulated using the extended simple point charge intermolecular potential. J. Chem. Phys. 107, 7443-7450 (1997)

38. Starr, F. W., Sciortino, F. \& Stanley, H. E. Dynamics of simulated water under pressure. Phys. Rev. E 60, 6757-6768 (1999).

39. Scala, A., Starr, F. W., La Nave, E., Stanley, H. E. \& Sciortino, F. The free energy surface of supercooled water. Phys. Rev. E 62, 8016-8020 (2000).

40. Poole, P. H., Essmann, U., Sciortino, F. \& Stanley, H. E. Phase diagram for amorphous solid water. Phys. Rev. E 48, 4605-4610 (1993).

41. Starr, F. W., Bellissent-Funel, M.-C. \& Stanley, H. E. Structure of supercooled and glassy water under pressure. Phys. Rev. E 60, 1084-1087 (1999).

42. Giovambattista, N., Stanley, H. E. \& Sciortino, F. A Potential Energy Landscape Study of the Amorphous-Amorphous Transformation in $\mathrm{H}_{2} \mathrm{O}$. Phys. Rev. Lett. 91, 115504 (2003).

43. Giovambattista, N., Stanley, H. E. \& Sciortino, F. Relation between the highdensity phase and the very high-density phase of amorphous solid water. Phys. Rev. Lett. 94, 107803 (2005).

44. Giovambattista, N., Stanley, H. \& Sciortino, F. Phase diagram of amorphous solid water: Low-density, high-density, and very-high-density amorphous ices. Phys. Rev. E 72 (2005)

45. Giovambattista, N., Debenedetti, P., Sciortino, F. \& Stanley, H. Structural order in glassy water. Phys. Rev. E 71 (2005)
46. Mishima, O., Calvert, L. D. \& Whalley, E. 'Melting ice' I at $77 \mathrm{~K}$ and 10 kbar: a new method of making amorphous solids. Nature 310, 393-395 (1984).

47. Hansen, J. P. \& McDonald, I. R. Theory of Simple Liquids (Academic Press, London, 1986).

48. Angell, C. A. Formation of glasses from liquids and biopolymers. Science 267, 1924-1935 (1995).

49. Errington, J. \& Debenedetti, P. G. Relationship between structural order and the anomalies of liquid water. Nature 409, 318-321 (2001).

50. Poole, P. H., Becker, S. R., Sciortino, F. \& Starr, F. W. Dynamical behavior near a liquid-liquid phase transition in simulations of supercooled water. J. Phys. Chem. B 115, 14176-14183 (2011).

51. Nelmes, R. J. et al. Annealed high-density amorphous ice under pressure. Nat. Phys. 2, 414-418 (2006).

52. Elsaesser, M. S., Winkel, K., Mayer, E. \& Loerting, T. Reversibility and isotope effect of the calorimetric glass $\rightarrow$ liquid transition of low-density amorphous ice. Phys. Chem. Chem. Phys. 12, 708-712 (2010).

53. Handa, Y., Mishima, O. \& Whalley, E. High Density Amorphous ice III. Thermal Properties. J. Chem. Phys. 84, 2766-2770 (1986)

54. Seidl, M., Loerting, T. \& Zifferer, G. High-density amorphous ice: Molecular dynamics simulations of the glass transition at $0.3 \mathrm{GPa}$. J. Chem. Phys. 131, 114502 (2009).

55. Prielmeier, F. X., Lang, E. W., Speedy, R. J. \& Lüdemann, H.-D. The pressure dependence of self-diffusion in supercooled light and heavy water. Ber. Bunsenges. Phys. Chem. 92, 1111 (1988).

56. Berendsen, H. J. C., Grigera, J. R. \& Stroatsma, T. P. The missing term in effective pair potentials. J. Phys. Chem. 91, 6269-6271 (1987).

57. Stillinger, F. H. \& Rahman, A. Improved simulation of liquid water by molecular dynamics. J. Chem. Phys. 60, 1545-1557 (1974).

58. Berendsen, H. J. C., Postma, J. P. M., Gunsteren, W. F. V., Dinola, A. \& Haak, J. R. Molecular dynamics with coupling to an external bath. J. Chem. Phys. 81, 3684 (1984).

59. Rahman, A. \& Stillinger, F. H. Proton distribution in ice and kirkwood correlation factor. J. Chem. Phys. 57, 4009-4017 (1972).

60. Winkel, K. et al. In Kuhs, W. F. (ed.) Physics and Chemistry of Ice chap. Isothermal amorphous-amorphous-amorphous transitions in water, 641-648 (The Royal Society of Chemistry, Cambridge, 2007).

\section{Acknowledgements}

Support of this project was provided by a PSC-CUNY Award to NG, jointly funded by The Professional Staff Congress and The City University of New York. TL is grateful for financial support by the Austrian Science Fund FWF (START award Y391) and the European Research Council (ERC) from grant SULIWA. FWS thanks the NSF for support from grant number CNS-0959856.

\section{Author contributions}

NG, TL, FS designed research and wrote manuscript. All authors performed research and reviewed the manuscript.

\section{Additional information}

Supplementary information accompanies this paper at http://www.nature.com/ scientificreports

Competing financial interests: The authors declare no competing financial interests.

License: This work is licensed under a Creative Commons

Attribution-NonCommercial-ShareAlike 3.0 Unported License. To view a copy of this license, visit http://creativecommons.org/licenses/by-nc-sa/3.0/

How to cite this article: Giovambattista, N., Loerting, T., Lukanov, B.R. \& Starr, F.W. Interplay of the Glass Transition and the Liquid-Liquid Phase Transition in Water. Sci. Rep. 2, 390; DOI:10.1038/srep00390 (2012). 